\title{
Identification of elasto-viscoplastic material properties from indentation testing using an inverse method
}

\author{
G. Rauchs \& J. Bardon \\ Laboratoire de Technologies Industrielles, \\ Centre de Recherche Public Henri Tudor, Grand Duchy of Luxembourg
}

\begin{abstract}
A combination of finite element modelling with gradient-based numerical optimization is used to determine the material parameters of a mechanical constitutive law from indentation testing by minimizing a cost function, quantifying the difference between load versus displacement-into-surface curves obtained from experiments and from finite element modelling. The required gradients of the cost function are calculated through a sensitivity analysis based on the direct differentiation method. Approximate correlation or cosine matrices will be calculated for assessing parameter correlation and used for designing load histories, which improve the parameter correlation in indentation testing. Finally, experimental indentation curves from metallic materials will be analyzed using the inverse method. The impact on material parameter identification of indenter tip size and of the incorporation of residual imprint data, obtained by white light interferometry, into the objective function will be monitored.
\end{abstract}

Keywords: indentation testing, finite element modelling, numerical optimization.

\section{Introduction}

Depth-sensing indentation and nanoindentation testing is being used for determining some material properties, such as Young's modulus and hardness following methods developed by Oliver and Pharr [1]. A rigid indenter is pushed into a specimen and the displacement-into-surface is recorded as a function of the applied load. Computational inverse methods have been developed for material parameter identification, either based on neural networks (Huber and 
Tsakmakis [2]) multiple response surface analysis (Hamasaki et al. [3]) or on numerical optimization (Constantinescu and Tardieu [4], Bolzon et al. [5], Rauchs [6]), which offer the possibility to determine material parameters of mechanical constitutive laws in addition to those found through standard experimental procedures. The inverse methods may be used in combination with the finite element method for determining material parameters, both for the case of simple tests, such as uniaxial tension (Mahnken and Stein [7]), and especially in the case of highly inhomogeneous, multiaxial stress fields, such as the indentation test or in the case of local damaging of the specimen. In optimization-based methods, the material parameters are determined by minimizing an objective function, quantifying the difference between load versus displacement-into-surface curves obtained from experiments and from finite element modelling. Most often, gradient-based optimization algorithms are used because they offer the potential for fast parameter identification, and the required gradients of the cost function can easily be calculated in a fast and reliable way through a sensitivity analysis based on either direct differentiation or adjoint-state methods. Some material parameters are difficult to determine, such as viscosity parameters (Constantinescu and Tardieu [4], Klötzer et al. [8]), or the separation of isotropic and kinematic hardening (Huber and Tsakmakis [2]) and the reliability of the results is poor because of strong parameter correlation. This stems from the fact that in indentation tests, some physical phenomena inherent to the constitutive laws may not affect the experimental curves in a significant or distinguishable way, as shown in Chen et al. [9]. In addition to this, the experimental data resulting from indentation testing, i.e. load and displacement-into-surface, are integrals of stress and strain, respectively, whereas the material constitutive law is based directly on stress and strain. In the present paper, the spherical indentation test is modelled by an axisymmetric finite element model for obtaining the objective function and the sensitivities with respect to the material parameters necessary for the material parameter identification algorithm. After presenting the material constitutive law, the computational framework and the sensitivity analysis based on direct differentiation, the investigation of material parameters of the aluminium alloy Dural will be presented, and the influence of experimental residual imprint measurements on parameter identification assessed.

\section{Elasto-viscoplastic law}

In the following, tensors, matrices and vectors are characterized by boldface letters, unless index notation is required for a clearer representation. A neo-Hookean hyperelastic law is used, which relates the Kirchhoff stress $\mathbf{S}$ to the left Cauchy-Green elastic deformation tensor $\mathbf{B}_{\mathrm{e}}$, defined through the elastic deformation gradient $\mathbf{F}_{\mathrm{e}}$, obtained from the standard multiplicative elastic-plastic decomposition of the deformation tensor $\mathbf{F}$. The hyperelastic constitutive law, with the elastic Lamé constants $v$ and $\lambda$, the determinant of the elastic deformation gradient $\mathbf{J}$ and the second order identity tensor $\mathbf{I}$ reads 


$$
\mathbf{S}=\mu\left(\mathbf{B}_{e}-\mathbf{I}\right)+\lambda \ln J \mathbf{I} .
$$

Plastic hardening with an associative flow rule is considered, including nonlinear isotropic and nonlinear kinematic hardening. The evolution of the yield limit $\mathrm{K}_{\mathrm{y}}$ is described through a non-linear isotropic hardening law, with

$$
K^{y}=\sqrt{\frac{2}{3}}(Y+R(1-\exp (-\beta S)))
$$

where $\mathrm{s}$ is the plastic arc length, $\mathrm{Y}$ is the initial uniaxial yield stress, and $\mathrm{R}$ and $\beta$ are the nonlinear isotropic hardening parameters. The back-stress, $\alpha$, and the plastic strain, $\boldsymbol{\varepsilon}_{\mathrm{p}}$, are related through the kinematic hardening evolution using the Armstrong-Frederick law with the non-linear kinematic hardening parameters $\mathrm{H}_{\text {kin }}$ and $\mathrm{b}$.

$$
\dot{\boldsymbol{\alpha}}=\sqrt{\frac{2}{3}} H_{k i n} b \dot{\boldsymbol{\varepsilon}}_{p}-b \dot{\boldsymbol{s}} \boldsymbol{\alpha} .
$$

In order to include viscoplasticity into the constitutive equations in a simple and efficient manner, the yield condition differs from the consistency condition. The yield condition $\mathrm{f}$ reads, with the deviatoric projection operator $\mathbf{P}$,

$$
f=\|\mathbf{P}:(\mathbf{S}-\boldsymbol{\alpha})\|-K^{y},\left\{\begin{array}{c}
f<0, \\
f \geq 0, \quad \text { elastic } \\
\text { viscoplastic }
\end{array},\right.
$$

For viscoplasticity, the consistency condition reads

$$
f^{\prime}=\|\mathbf{P}:(\mathbf{S}-\boldsymbol{\alpha})\|-K^{y}-\sqrt{\frac{2}{3}}(\dot{s} \eta)^{\frac{1}{m}},
$$

where $\eta$ is the viscosity and $m$ is the viscosity exponent.

\section{Stress update equations}

In the following, the state at the beginning of the time increment is characterized by the prefix ${ }^{0}$, the state at the end of the increment by ${ }^{1}$. The time integration of the constitutive equation is performed through an operator split: an elastic predictor step followed by an inelastic corrector step. In the elastic predictor step, no inelastic deformation takes place and the deformation gradient $\mathbf{F}$ is modified by $\Delta \mathbf{F}$, whereas in the inelastic corrector step the deformation gradient $\mathbf{F}$ remains unchanged and inelastic deformation takes place. The elastic predictor step gives the trial state, labelled by the prefix ${ }^{\operatorname{Tr}}$,

$$
{ }^{T r} \mathbf{B}_{e}=\Delta \mathbf{F}^{0} \mathbf{B}_{e} \Delta \mathbf{F}^{T},
$$

with

$$
\Delta \mathbf{F}={ }^{1} \mathbf{F}^{0} \mathbf{F}^{-1}
$$


The predictor of the back-stress of the elasto-viscoplastic constitutive law is calculated similarly to eqn (6). The inelastic corrector leads to the stress and internal variable update equations used in the direct deformation finite element code. In order to simplify the notations, we introduce a generalized directional derivative, similar to a Gateaux-differential, which designates derivatives with respect to time or with respect to a material parameter $\kappa$. Such a differential of a tensor $\mathbf{X}$ is written as

$$
[\mathbf{X}]^{\diamond}
$$

The linearization of the Kirchhoff stress at the end of the increment gives a relationship in the following form

$$
\left[{ }^{1} \mathbf{S}\right]^{\diamond}=\partial^{1} \mathbf{S}+\mathbf{D}^{m a t}:\left[\frac{\partial \Delta \mathbf{u}}{\partial^{1} \mathbf{x}}\right]^{\diamond}
$$

where $\mathbf{D}^{\text {mat }}$ is the consistent constitutive tensor, derived from the stress update equations. The first term on the right hand side regroups the explicit sensitivities of the material parameter and the sensitivities of the stress at the beginning of the increment. It is important to notice that this term is only active in the sensitivity analysis and vanishes for the linearization used in the Newton-Raphson iteration, which is a linearization with respect to the displacement increment, $\Delta \mathbf{u}$, upon which the state variables at the increment start do not depend.

\section{Sensitivity analysis}

Sensitivity analysis is performed using the direct differentiation method, which is presented in Rauchs [6], for example, because of its conceptual simplicity. Starting from the basic equilibrium equation of non-linear finite element analysis and using the element shape function $\mathrm{N}^{\mathrm{A}}$, the current position vector ${ }^{1} \mathbf{x}$ and integrating over the body in the current configuration, the discretized form is obtained:

$$
\int_{\Omega} \frac{\partial N^{A}}{\partial^{1} \mathbf{x}}{ }^{1} \boldsymbol{\sigma} d \Omega-\mathbf{f}^{A, e x t}-\mathbf{f}^{A, c o n}=0,
$$

where $\mathbf{f}^{\mathrm{A}, \mathrm{ext}}$ is the external nodal load and $\mathbf{f}^{\mathrm{A}, \mathrm{con}}$ is the contact nodal load at nodes on the contact boundary. The derivative of eqn (10) with respect to a material parameter $\kappa$ yields

$$
\left[\int_{\Omega} \frac{\partial N^{A}}{\partial^{1} \mathbf{x}} \boldsymbol{\sigma} d \Omega\right]^{\ominus}-\left[\mathbf{f}^{A, c o n}\right]^{\ominus}=0 .
$$

In case of conservative external nodal loads, the sensitivity of the second term in eqn (10) vanishes. Replacing the Cauchy stress, $\sigma$, by the Kirchhoff stress, $\mathbf{S}$, using the sensitivity of the Kirchhoff stress specific to the constitutive law, 
eqn (9), and performing some lengthy mathematical developments, which can be found in Rauchs [6], for example, eqn (11) becomes

$$
\begin{gathered}
\int_{\Omega} \frac{\partial N^{A}}{\partial^{1} \mathbf{x}}\left(-\left[\frac{\partial \Delta \mathbf{u}}{\partial^{1} \mathbf{x}}\right]^{\diamond} \frac{{ }^{1} \mathbf{S}}{{ }^{1} J}+\mathbf{D}^{m a t}:\left[\frac{\partial \Delta \mathbf{u}}{\partial^{1} \mathbf{x}}\right]^{\diamond}\right) d \Omega \\
+\int_{\Omega} \frac{\partial N^{A}}{\partial^{1} \mathbf{x}}\left(-\left[{ }^{0} \mathbf{F}\right]^{{ }^{1}} \mathbf{F}^{-1}{ }^{1} \mathbf{S}+\partial^{1} \mathbf{S}\right) d \Omega+\left[\mathbf{f}^{A, c o n}\right]^{\diamond}=0
\end{gathered}
$$

where the first integral contains terms depending on the sensitivity of the displacement increment and the second integral contains only terms known at the beginning of the increment. With the sensitivity of the displacement gradient interpolated through

$$
\left[\frac{\partial \Delta u_{m}}{\partial^{1} x_{n}}\right]^{\diamond}=\frac{\partial N^{B}}{\partial^{1} x_{n}}\left[\Delta u_{m}^{B}\right]^{\diamond},
$$

the two integrals can be further condensed into

$$
\left(\mathbf{K}^{A B}+\mathbf{K}^{A B, c o n}\right)\left[\Delta \mathbf{u}^{B}\right]^{\triangleright}+{ }^{\kappa} \mathbf{V}^{A}=0,
$$

with

$$
K_{i m}^{A B}=\int_{\Omega} \frac{\partial N^{A}}{\partial^{1} x_{j}}\left(D_{i j m n}^{m a t}-I_{m j} \frac{{ }^{1} S_{i n}}{{ }^{1} J}\right) \frac{\partial N^{B}}{\partial^{1} x_{n}} d \Omega
$$

and

$$
{ }^{\kappa} \mathbf{V}^{A}=\int_{\Omega} \frac{\partial N^{A}}{\partial^{1} \mathbf{x}}\left(-\left[{ }^{0} \mathbf{F}\right]^{{ }^{1} 1} \mathbf{F}^{-11} \mathbf{S}+\partial^{1} \mathbf{S}\right) d \Omega+\mathbf{K}^{A B, c o n}\left[{ }^{0} \mathbf{x}\right],
$$

in which repeated capital indices stand for the summation over all the nodes coupled to node A through solid continuum elements. $\mathbf{K}^{\mathrm{AB}, \mathrm{con}}$ is the contact stiffness matrix. In the frictionless case considered here, the contact force sensitivity depends on geometric quantities only. It should be noted that the differential of ${ }^{0} \mathbf{x}$ is known from the previous increment or the boundary conditions, respectively. Therefore, ${ }^{\mathrm{K}} \mathbf{V}^{\mathrm{A}}$ contains only known entities and can be calculated explicitly. By summing up eqns (15) and (16) over all connected nodes, the global stiffness matrix and pseudo-load vector can be constructed.

After resolving the sensitivities of the displacement increment, $\Delta \mathbf{u}$, the update of the sensitivities of the state variables is done in a postprocessing step. As the use of Oldroyd rates in the constitutive formulation gives a compute-efficient fully consistent linearization of the stress update equations, the same stiffness matrices may be used in direct deformation modelling and in the direct differentiation sensitivity analysis. Therefore, the latter one is performed at the end of each time increment, after convergence of the non-linear state variable update through the Newton-Raphson iteration. In the direct differentiation calculation, each increment is treated in three stages:

1. Calculation of the pseudo-stiffness matrix and pseudo-load vector from eqns. (14) and (16). 
2. Solution of eqn (14), which is a linear equation, for each material parameter to obtain the unknown displacement increment derivatives.

3. Back substitution of the displacement increment derivatives to obtain the updated derivatives of geometry and the state variables at the end of the increment.

\section{Numerical optimization procedure}

For the indentation test, the objective function to be minimized by a numerical optimization algorithm is made up of the difference between the experimental and modelled displacement-into-surface, $\mathrm{h}$ and $\mathrm{h}$,, respectively, which are a function of the independent load $\mathrm{P}$ :

$$
\Xi=\frac{1}{2} \sum_{k=1}^{N}\left[h\left(P^{k}\right)-h^{\prime}\left(P^{k}\right)\right]^{2} \text {. }
$$

A gradient-based optimization algorithm requires the derivative of the objective function with respect to a material parameter $\kappa$, given as

$$
\frac{\partial \Xi}{\partial \kappa_{i}}=\sum_{k=1}^{N}\left[h\left(P^{k}\right)-h^{\prime}\left(P^{k}\right)\right] \frac{\partial h\left(P^{k}\right)}{\partial \kappa_{i}} .
$$

The Hessian matrix is then

$$
\frac{\partial^{2} \Xi}{\partial \kappa_{i} \partial \kappa_{j}}=\sum_{k=1}^{N}\left[\frac{\partial h\left(P^{k}\right)}{\partial \kappa_{i}} \frac{\partial h\left(P^{k}\right)}{\partial \kappa_{j}}+\left[h\left(P^{k}\right)-h^{\prime}\left(P^{k}\right)\right] \frac{\partial^{2} h\left(P^{k}\right)}{\partial \kappa_{i} \partial \kappa_{j}}\right] .
$$

As the second term of the sum is known to adversely affect convergence, this term is neglected, and the approximated Hessian labelled as $H^{\prime}$. The minimization algorithm iteratively calculates the material parameters using standard Gauss-Newton algorithm. Additional experimental data can be included through mapping the residual imprint remaining after complete load removal, Bolzon et al. [5]. The objective function will be extended by a term comprising the differences of experimental and modelled imprint. For quantifying the residual imprint, the total vertical displacement, $\mathrm{h}^{\text {res }}\left(\mathrm{r}^{\mathrm{l}}\right)$ of the contact surface at a set of $\mathrm{M}$ fixed radial locations $r$, with respect to a chosen reference point, for example the imprint centre, called $\mathrm{r}^{0}$, is used, and an aggregate objective function takes the form:

$$
\Xi^{\prime}=\frac{1}{N} \Xi+\frac{1}{2 M} \sum_{k=1}^{M}\left[\left(h_{r e s}^{\prime}\left(r^{l}\right)-h_{r e s}^{\prime}\left(r^{0}\right)\right)-\left(h_{r e s}\left(r^{l}\right)-h_{r e s}\left(r^{0}\right)\right)\right]^{2} \text {. }
$$

In practice, the state variable history is calculated by solving the direct deformation problem using the finite element method. Subsequently, the derivative of $h$ is calculated by linear update, as described above. The objective function minimization is performed through the overall optimization loop. In the present study, the system response considered is the displacement-into-surface, which has the same order of magnitude as the residual imprint. Therefore, no scaling factor needs to be introduced in eqn (20), apart from the scaling with respect to the number of data available for each term, $\mathrm{N}$ and $\mathrm{M}$. 
From uniaxial tension/compression tests for material parameter identification of elasto-plastic materials in Mahnken and Stein [7], it was found that the compressive cycle is necessary for uniquely determining isotropic and kinematic hardening parameters. In the absence of a compressive cycle with reverse plastic flow, it is not possible to separate kinematic from isotropic hardening. In case the Hessian matrix is available, correlation or cosine matrices can be calculated and used to visualize the material parameter correlation. The cosine matrix indicates if the different sensitivities are co-linear. Even if the approximated Hessian matrix in eqn (19) does not include terms with the second order derivatives, it can give an indication of the parameter correlation. In fact, $\mathrm{H}^{\prime}$ is equivalent to the Hessian matrix if the objective function is exactly zero and as such it should give a sufficient information about parameter correlation in indentation testing. The cosine matrix of the Hessian matrix in eqn (19) is defined according to Forestier et al. [10], without summation over double indices, as

$$
c_{i j}=\frac{H_{i j}^{\prime}}{\sqrt{H_{i i}^{\prime} H_{j j}^{\prime}}} .
$$

The total correlation between material parameters is quantified by the correlation factor, $\mathrm{k}$, defined as

$$
k=\sqrt[72]{\prod_{i, j=1, i \neq j}^{9} c_{i j}} .
$$

\section{Finite element model}

The finite element model of the indentation test consists of an axisymmetric model discretizing both the indenter and the specimen as flexible bodies. The spherical indenter has a tip radius of $5 \mu \mathrm{m}$ and the overall shape is a cone with an opening angle of 60 degrees. Quadratic elements with displacement degrees of freedom have been used. Contact constraints are fulfilled by using $\mathrm{C}^{2}$-continuous quadratic node-to-segment contact elements with a modified $\mathrm{C}^{2}$-continuous logarithmic barrier potential according to Kloosterman [11]. Instead of an augmented Lagrangian contact algorithm, a similar algorithm with intermediate loops has been used, where the penalty parameter is increased from one intermediate loop to the next. The motivation is to have an explicit form of the contact tractions, unlike the augmented Lagrangian algorithm, where the contact tractions are calculated iteratively in the intermediate loops, which then have to be performed in the sensitivity analysis as well. At the end of each intermediate loop, an extrapolation according to Dussault [12] is performed in order to improve convergence. A line search method is included into the NewtonRaphson iteration loop in order to improve overall convergence.

The finite element calculations are performed under load control, because most nanoindenters operate in this mode and the noise on load is usually smaller than that on the displacement measurements. Computations of the indentation test showed that at indentation loads, mesh locking becomes an issue. This 
problem was overcome by the use of nine-noded fully integrated quadratic elements. However, it should be noticed that such elements require larger computing times.

\section{Results}

In the first place, the correlation factor, $\mathrm{k}$, has been calculated to assess parameter correlation in parameter identification based on nanoindentation testing. It was found that a hold period is crucial for reducing the parameter coupling between viscosity parameters on the one hand and elastic and plastic material parameters on the other hand, reducing $\mathrm{k}$ from 0.98 to 0.66 for a single load cycle. Inclusion of residual imprint data, according to eqn (20), provided some decoupling between the elastic parameter and the plastic parameters, reducing $\mathrm{k}$ to 0.52 . However, the parameters within these three groups of material parameters, i.e. elastic, plastic and viscous, remained nearly fully coupled. It was somewhat striking to note that with the hysteresis produced during reloading in a second load cycle, no uncoupling between isotropic and kinematic hardening parameters was monitored, whereas in Huber and Tsakmakis [2], this hysteresis can be used successfully for parameter identification in neural network-based parameter identification.

In a last stage, material parameter identification was performed using true experimental nanoindentation curves. The material under investigation was an aluminium alloy, Dural, where unfortunately only a limited number of material parameters from tensile testing is available. Three indenter tip radii where used: $5 \mu \mathrm{m}, 10 \mu \mathrm{m}$ and $50 \mu \mathrm{m}$, and the indenters are labelled R5, R10 and R50, respectively. It should be noted that these are nominal radii. In the finite element models, measured radii have been used.

Table 1: $\quad$ Material parameters identified from first load cycle.

\begin{tabular}{|l|r|r|r|r|r|r|}
\hline Parameter & R5 & R5 err & R10 & R10 err & R50 & R50 err \\
\hline E $(\mathrm{MPa})$ & 60462 & $3.8 \%$ & 72634 & $4.0 \%$ & 80860 & $3.2 \%$ \\
\hline$\nu(-)$ & 0.37 & $9.7 \%$ & 0.24 & $11.9 \%$ & 0.40 & $1.7 \%$ \\
\hline $\mathrm{Y}(\mathrm{MPa})$ & 228 & $12.6 \%$ & 77 & $67.3 \%$ & 745 & $6.6 \%$ \\
\hline $\mathrm{R}(\mathrm{MPa})$ & 143 & $24.7 \%$ & 673 & $17.0 \%$ & 189 & $79.2 \%$ \\
\hline$\beta(-)$ & 28.0 & $5.2 \%$ & 7.1 & $9.4 \%$ & 7.8 & $49.0 \%$ \\
\hline $\mathrm{H}_{\mathrm{kin}}(\mathrm{MPa})$ & 169 & $5.0 \%$ & 479 & $14.3 \%$ & 741 & $120.2 \%$ \\
\hline $\mathrm{b}(-)$ & 31.2 & $15.4 \%$ & 7.4 & $9.9 \%$ & 8.5 & $40.1 \%$ \\
\hline$\eta(\mathrm{MPa} \mathrm{s})^{1 / \mathrm{m}}$ & $1.6 \mathrm{E}+08$ & $146.7 \%$ & $7.0 \mathrm{E}+06$ & $67.3 \%$ & $1.9 \mathrm{E}+07$ & $39.6 \%$ \\
\hline $\mathrm{m}(-)$ & 4.3 & $15.6 \%$ & 3.2 & $6.8 \%$ & 2.0 & $12.6 \%$ \\
\hline$\sum(\mathrm{MPa})$ & 624 & $1.2 \%$ & 1468 & $11.8 \%$ & 2045 & $70.4 \%$ \\
\hline
\end{tabular}

In table 1 , the material parameters identified from input data made up of the first load cycle only are given. A comparison with the only reliable material parameter available, the Young's modulus of $73000 \mathrm{MPa}$, shows that the indenters R5 and R50 give erroneous results whereas indenter R10 gives a very 
accurate value of the Young's modulus. Residual imprint measurements indicate that the shapes of the indenters R5 and R50 significantly deviate from the assumed spherical shape. The Poisson's ratio, assumed to be around 0.3 for metals, is also determined quite reliably. However, the indenter R50 gives poor values for the plastic material parameters, as even the yield stress Y lies above the strength of Dural, which is approximately $500 \mathrm{MPa}$. It can be realized that $\Sigma$, the weighted sum of $\mathrm{Y}, \mathrm{R}$ and $\mathrm{H}_{\text {kin }}$, representing the maximum hardening stress, is identified with very little scatter. The hardening exponents $\beta$ and $b$ are high for all indenters, especially for R5, and will produce nearly full hardening after only small amounts of plastic deformation. The viscosity $\eta$ exhibits large scatter and is therefore unreliable. In fact, as viscosity plays a role in indentation testing because of the high compressive stresses occurring in indentation testing, the viscosity parameter should be considered as no more than numerical parameters used for introducing the right amount of creep deformation found in indentation testing, but irrelevant in room temperature applications.

Table 2: $\quad$ Material parameters identified from the two-cycled load history.

\begin{tabular}{|l|r|r|r|r|r|r|}
\hline Parameter & R5 & R5 err & R10 & R10 err & R50 & R50 err \\
\hline E $(\mathrm{MPa})$ & 58593 & $2.9 \%$ & 72281 & $3.4 \%$ & 70812 & $1.1 \%$ \\
\hline$\nu(-)$ & 0.38 & $28.1 \%$ & 0.25 & $14.8 \%$ & 0.49 & $0.3 \%$ \\
\hline $\mathrm{Y}(\mathrm{MPa})$ & 325 & $7.7 \%$ & 66 & $58.9 \%$ & 688 & $6.2 \%$ \\
\hline $\mathrm{R}(\mathrm{MPa})$ & 113 & $14.6 \%$ & 739 & $1.7 \%$ & 33 & $132.2 \%$ \\
\hline$\beta(-)$ & 15.6 & $19.8 \%$ & 6.7 & $27.4 \%$ & 5.6 & $53.7 \%$ \\
\hline $\mathrm{H}_{\text {kin }}(\mathrm{MPa})$ & 126 & $22.8 \%$ & 488 & $6.6 \%$ & 52 & $80.5 \%$ \\
\hline $\mathrm{b}(-)$ & 18.8 & $28.5 \%$ & 7.4 & $21.0 \%$ & 5.2 & $52.2 \%$ \\
\hline$\eta(\mathrm{MPa} \mathrm{s})^{1 / \mathrm{m}}$ & $3.3 \mathrm{E}+07$ & $88.4 \%$ & $9.7 \mathrm{E}+06$ & $37.8 \%$ & $7.6 \mathrm{E}+04$ & $77.8 \%$ \\
\hline $\mathrm{m}(-)$ & 4.3 & $12.8 \%$ & 3.1 & $6.1 \%$ & 4.0 & $64.7 \%$ \\
\hline \hline$\Sigma(\mathrm{MPa})$ & 627 & $2.7 \%$ & 1536 & $5.4 \%$ & 799 & $7.7 \%$ \\
\hline
\end{tabular}

Table 3: $\quad$ Material parameters identified from the two-cycled indentation test, with and without residual imprint data, for nominal tip radius of $10 \mu \mathrm{m}$.

\begin{tabular}{|l|r|r|r|r|}
\hline Parameter & R10i & R10i err & R10 & R10 err \\
\hline E $(\mathrm{MPa})$ & 45924 & $7.5 \%$ & 72281 & $3.4 \%$ \\
\hline$\nu(-)$ & 0.39 & $20.0 \%$ & 0.25 & $14.8 \%$ \\
\hline $\mathrm{Y}(\mathrm{MPa})$ & 507 & $2.6 \%$ & 66 & $58.9 \%$ \\
\hline $\mathrm{R}(\mathrm{MPa})$ & 439 & $23.7 \%$ & 739 & $1.7 \%$ \\
\hline$\beta(-)$ & 1.1 & $7.2 \%$ & 6.7 & $27.4 \%$ \\
\hline $\mathrm{H}_{\text {kin }}(\mathrm{MPa})$ & 400 & $31.7 \%$ & 488 & $6.6 \%$ \\
\hline $\mathrm{b}(-)$ & 1.6 & $24.5 \%$ & 7.4 & $21.0 \%$ \\
\hline$\eta(\mathrm{MPa} \mathrm{s})^{1 / \mathrm{m}}$ & $1.7 \mathrm{E}+06$ & $56.7 \%$ & $9.7 \mathrm{E}+06$ & $37.8 \%$ \\
\hline $\mathrm{m}(-)$ & 6.8 & $25.5 \%$ & 3.1 & $6.1 \%$ \\
\hline \hline$\sum(\mathrm{MPa})$ & 1546 & $10.0 \%$ & 1536 & $5.4 \%$ \\
\hline
\end{tabular}




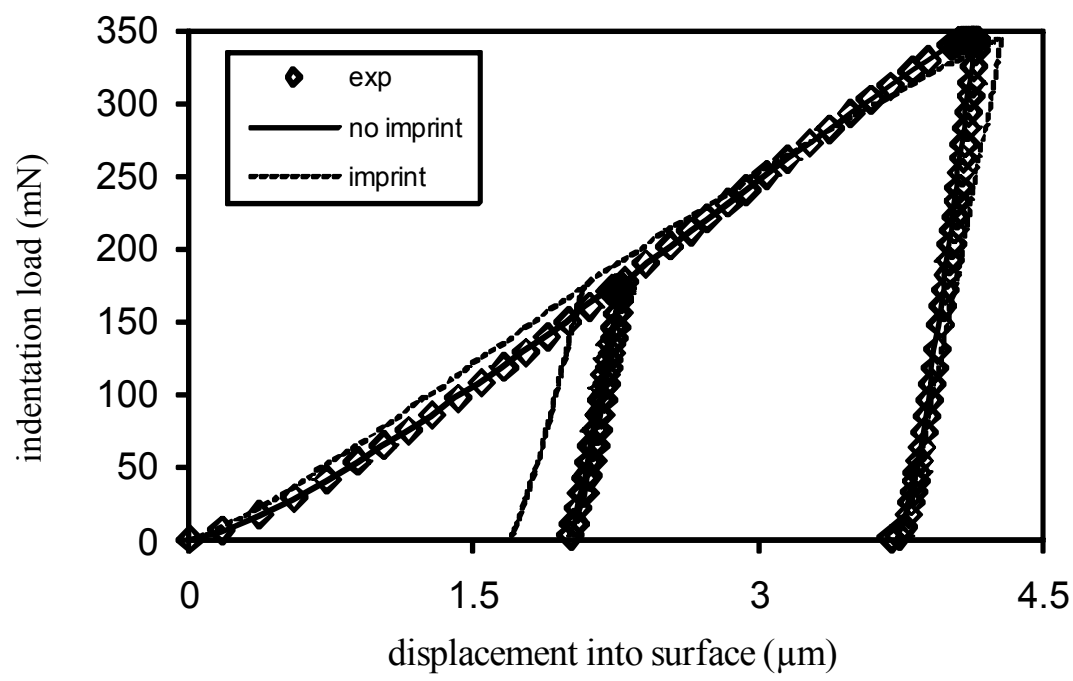

Figure 1: Indentation curves for indenter tip radius of $10 \mu \mathrm{m}$ : experimental (symbols), computed from parameters identified from load curves only (solid line) and from load curve and imprint data (dotted line).

In table 2, material parameters identified from the full load history comprising two consecutive load cycles, with the second peak load being twice the first load peak, are shown. No major improvement over the parameters presented in table 1 are visible, with the largest change being found in the viscosity. A more prominent change in material parameters takes place if residual imprint data are included into the objective function according to eqn (20), see table 3 . Here, one can see that on the one hand, the Young's modulus deteriorates, whereas the yield stress increases. The hardening exponents $\beta$ and $b$ are also much lower. It can be seen in Fig. 1 that the indentation curve obtained from the parameters identified with residual imprint data does not fit well with the experimental indentation curves, unlike the indentation curves obtained from indentation curve data only.

The situation is completely different for the modelled residual imprint, where pile-up takes place for experimental input including residual imprint measurements, whereas sink-in occurs in the absence of imprint data, which is in contradiction to the experimental residual imprint data, as depicted in Fig.2. It is known that the ratio between yield stress and Young's modulus determines whether pile-up or sink-in occurs, which is a fundamental aspect of each specific material. This is a clear indication that the yield stress found without imprint data is much too low, the value determined with imprint data seems much more appropriate from the point of view of pile-up. However, this value lies above the strength given by the manufacturer. It should be noted that the residual imprint data of the centre of the imprint have been removed from the objective function 
because they do not fit the measured residual displacement into surface from the indentation curve. The absence of data points between 3 and $7 \mu \mathrm{m}$ in radial direction stems from the fact that white light interferometry does only yield data for surfaces whose inclination with respect to the horizontal lies below a magnification-dependent threshold, $25^{\circ}$ in our case, leading to a large blind spot in case of deep imprints. It has to be said that the residual imprint measurements are highly affected by experimental scatter, either caused by surface defects like scratches, or by polycrystalline anisotropic deformation taking place.

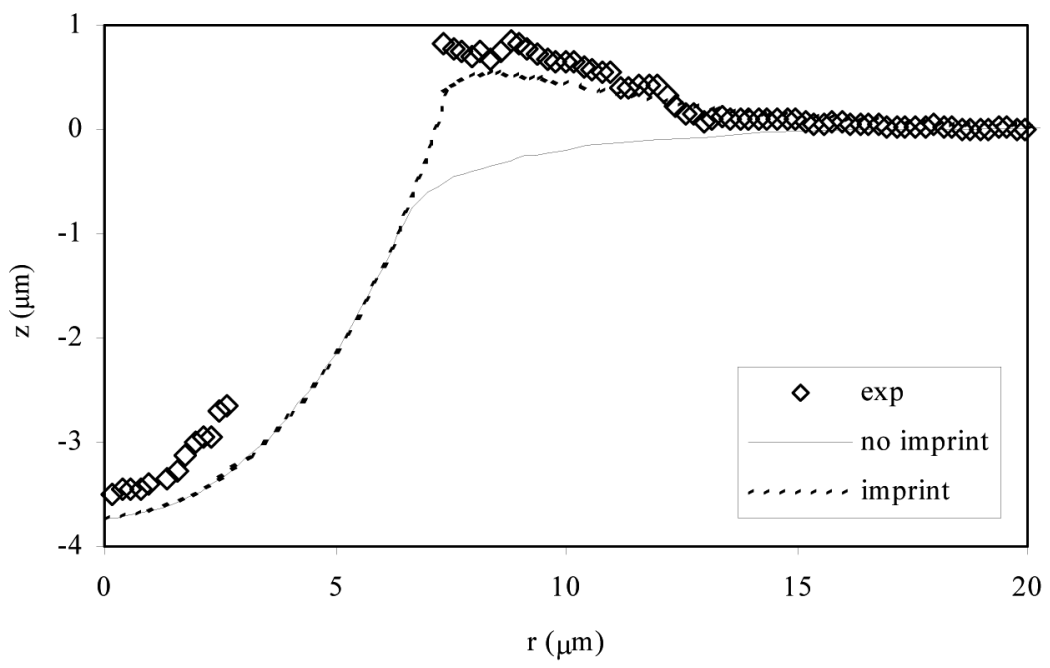

Figure 2: Residual imprint profiles for indenter tip radius of $10 \mu \mathrm{m}$ : experimental (symbols), computed from parameters identified from load curves only (solid line) and from load curve and imprint data (dotted line).

To resume, the identification of material parameters from nanoindentation testing coupled to an inverse method is not very reliable in the current form, with the exception of the Young's modulus, where quite reliable results with very low scatter may be obtained. Progress has to be made for determined the plastic material parameters, and a more efficient use of residual imprint data may be required for achieving more reliable results. Another issue is the influence of surface roughness on the indentation curves, as stated in Klötzer et al. [8], which has a significant impact on the initial part of the indentation curve, because surface asperities are less stiff than the modelled, perfectly flat specimen surface.

\section{References}

[1] Oliver, W.C. \& Pharr, G.M., An improved technique for determining hardness and elastic modulus using load and displacement sensing indentation experiments, J. Mater. Res. 7, pp. 1564-1583, 1992. 
[2] Huber, N. \& Tsakmakis, Ch., Determination of constitutive properties from spherical indentation data using neural networks. Part II: Plasticity with nonlinear isotropic and kinematic hardening. J. Mech. Phys. Solids, 47, pp. 1589-1607, 1999.

[3] Hamasaki, H., Toropov, V.V., Kazuhiro, K. \& Yoshida F., Identification of Viscoplastic Properties for Lead-Free Solder by Microindentation Experiments, FE Simulation and Optimization, J. Japan Soc. Tech. Plast. 46, pp. 397-401, 2005.

[4] Constantinescu, A. \& Tardieu, N., On the identification of elastoviscoplastic constitutive laws from indentation tests. Inverse Problems in Engineering, 9, pp. pp. 19-44, 2001.

[5] Bolzon, G., Maier, G. \& Panico, M., Material model calibration by indentation, imprint mapping and inverse analysis. Int. J. Sol. Struct., 41, pp. 2957-2975, 2004.

[6] Rauchs, G., Optimization-based material parameter identification in indentation testing for finite strain elasto-plasticity. ZAMM, 86, pp. 539562, 2006.

[7] Mahnken, R. \& Stein, E., Parameter identification for viscoplastic models based on analytical derivatives of a least-squares functional and stability investigations. Int. J. Plasticity, 12, pp. 451-479, 1996.

[8] Klötzer, D., Ullner, Ch., Tyulyukovskiy, E. \& Huber N., Identification of viscoplastic material parameters from spherical indentation data. Part II: Experimental validation of the method, J. Mater. Res. 21, pp. 677-684, 2006.

[9] Chen, X., Ogasawara, N., Zhao, M. \& N. Chiba, N., On the uniqueness of measuring elastoplastic properties from indentation: The indistinguishable mystical materials. J. Mech. Phys. Solids, 55, pp. 1618-1660, 2007.

[10] Forestier, R., Chastel, Y. \& Massoni, E., 3d inverse analysis model using semi-analytic differentiation for mechanical parameter estimation. Inverse Problems in Eng., 11, pp. 255-271, 2003.

[11] Kloosterman, G., Contact Methods in Finite Element Methods. PhD thesis, University of Twente, 2002.

[12] Dussault, J.-P., Numerical stability and efficiency of penalty algorithms. SIAM J. Num. Anal., 32, pp. 296-317, 1995. 